\title{
Association between miR-146a SNP rs2910164 and ischemic stroke in Asian population: a meta-analysis
}

\author{
Xue-qi Yang ${ }^{1}$ and Jiao Zhang ${ }^{2 *}$ \\ ${ }^{1}$ The Fifth Affiliated Hospital of Zhengzhou University, Zhengzhou 450052, China \\ ${ }^{2}$ Anatomy and Cell Biology, East Carolina University, Greenville, NC, 27834, USA
}

\begin{abstract}
The microRNA146a rs2910164 polymorphism has been associated with the development of ischemic stroke; however, the results were inconsistent among different studies. The present report was aimed to investigate the association between rs $2910164 \mathrm{G} / \mathrm{C}$ polymorphism and the risk of ischemic stroke. Several studies have been carried out to explore the association between this SNP and ischemic stroke in Asians, but published results were contradictory. In the present study, we performed a meta-analysis to further evaluate this association in Asian population. All relevant articles were retrieved from the databases of PubMed, EMBASE, CNKI, WANFANG Database and CQVIP from the establishment date to February 2015. Statistical analyses were performed by using Stata 11 . The pooled odds ratios (ORs) with 95\% confidence intervals (95\% CIs) were used to assess the strength of association. Possible publication bias was checked by funnel plots, Begg's test and Egger's test. Six studies were eligible for meta-analysis including 2242 cases and 2359 controls. Overall, there were no significant associations between rs2910164 and ischemic stroke under the allelic (OR=1.051, 95\%CI: 0.881, 1.254), dominant (OR=1.092, 95\% CI: 0.909, 1.311), recessive (OR=1.036, 95\%CI: 0.738, 1.454), homozygote $(\mathrm{OR}=1.080,95 \% \mathrm{CI}$ : $0.726,1.608)$ and heterozygote $(\mathrm{OR}=1.092,95 \% \mathrm{CI}: 0.961,1.242)$ genetic models in Asian population. Stratified analysis based on ethnicity shows that rs 2910164 has no association with ischemic stroke in Chinese population (all P>0.05), although this association exists in Korean. Our metaanalysis shows that the miR-146a SNP rs2910164 is not relative to ischemic stroke in Asian population, although it is a risk factor in Korean.
\end{abstract}

\section{Introduction}

Stroke is a major cause of disability and death in aging population and about $73 \%$ to $86 \%$ of strokes were ischemic $[1,2]$. Heritability for all ischemic stroke was 37.9\% [3]. MicroRNA, a class of small regulatory RNA, affects the cellular proteome and transcriptome through interacting with its mRNA targets at the $3^{\prime}$ untranslated region [4]. Recent studies showed that miRNAs play critical roles in modulating key biological processes involved in cell development, differentiation, growth, and metabolism [5]. Over the past few years several epidemiological studies were performed to evaluate the relation between the SNP rs2910164 and ischemic stroke in Asians, yet these research results failed to reach an agreement. For investigating the association between the SNP rs2910164 and ischemic stroke in Asian population, we performed this meta-analysis with new searchable data.

\section{Materials and methods}

\section{Search strategy}

We searched in the electronic databases of PubMed, EMBASE, CNKI, CQVIP and WANFANG Database from the establishment date to February 2015. The following search terms were used in isolation and combination with one another: "stroke" or "cerebral ischemic" or "cerebral infarction" combined with "rs2910164" or "microRNA-146a" or "miR-146a". All studies matching the eligible criteria were included in our meta-analysis. The reference lists of included articles were also reviewed for additional literature.

\section{Inclusion criteria}

The selected original studies should comply with the following inclusion criteria: a) concerning the association between rs2910164 and ischemic stroke risk; b) case-control studies, c) with full text; d) based on Asian population; e) sufficient genotype distribution data for estimating an odds ratio (OR) with $95 \%$ confidence interval (CI); f) exclusion of conference papers; g) genotype distribution of control population must be in Hardy-Weinberg equilibrium (HWE).

\section{Data extraction}

Two researchers read all publications complying with the inclusion criteria listed above and extracted the data independently. Disagreement was resolved through discussion. The following data were collected from each included study: first author's name, publication date, country, study design, total numbers of cases and controls and frequency of rs2910164 polymorphism in cases and controls.

\section{Statistical analysis}

Pearson's goodness-of-fit chi-square test was used to test the HWE for the genotype distributions in control group of each study. The heterogeneity was calculated by $\mathrm{Q}$ test and the inconsistency index $\left(\mathrm{I}^{2}\right)[6]$. The heterogeneity was considered significant when $\mathrm{P}<0.10$ or $\mathrm{I}^{2}>50 \%$ [6], and a random effects model was used, otherwise, a fixed effects model was adopted to calculate the pooled OR. The effect size was expressed as OR and 95\% CI. Five genetic models were used to

Correspondence to: Jiao ZHANG, Anatomy and Cell Biology, East Carolina University, Greenville, NC, 27834, USA; E-mail: zhangj15@ecu.edu

Key words: ischemic stroke, microRNA-146a, rs2910164, single nucleotide polymorphisms, meta-analysis

Received: August 05, 2015; Accepted: August 31, 2015; Published: September 05, 2015 
Table 1. Main characteristics and allele distributions of all studies included in the meta-analysis.

\begin{tabular}{|c|c|c|c|c|c|c|c|c|c|c|c|c|c|c|c|c|}
\hline \multirow[t]{2}{*}{ Study } & \multirow[t]{2}{*}{ Ethnicity } & \multirow[t]{2}{*}{ Control source } & \multirow{2}{*}{$\begin{array}{l}\text { Genotyping } \\
\text { method }\end{array}$} & \multicolumn{6}{|c|}{ IS patients } & \multirow[b]{2}{*}{ No. (Male \%) } & \multicolumn{6}{|c|}{ Controls } \\
\hline & & & & No.(Male \%) & $\mathrm{CC}$ & GC & GG & $\mathrm{C}$ & G & & $\mathrm{CC}$ & GC & GG & $\mathrm{C}$ & G & HWE $P$ \\
\hline Huang (2015) & Chinese & hospital-based & TaqMan & $531(61.6)$ & 189 & 261 & 81 & 639 & 423 & $531(61.6)$ & 219 & 257 & 55 & 695 & 367 & 0.106 \\
\hline Liu (2014) & Chinese & hospital-based & PCR-RFLP & $296(60.8)$ & 85 & 159 & 52 & 329 & 263 & $391(58.1)$ & 116 & 198 & 77 & 430 & 352 & 0.650 \\
\hline Zhu (2014) & Chinese & hospital-based & PCR-LDR & $368(68.8)$ & 145 & 173 & 50 & 463 & 273 & $381(68.5)$ & 132 & 185 & 64 & 449 & 313 & 0.952 \\
\hline $\mathrm{Hu}(2014)$ & Chinese & hospital-based & PCR-RFLP & $196(48.0)$ & 75 & 87 & 34 & 237 & 155 & $205(46.3)$ & 97 & 82 & 26 & 276 & 134 & 0.193 \\
\hline $\mathrm{Li}(2014)$ & Chinese & hospital-based & SNaPshot & $173(68.2)$ & 73 & 85 & 15 & 231 & 115 & $298(64.4)$ & 111 & 136 & 51 & 358 & 238 & 0.401 \\
\hline Jeon (2013) & Korean & hospital-based & PCR-RFLP & $678(49.6)$ & 223 & 327 & 128 & 773 & 583 & $553(44.1)$ & 211 & 266 & 76 & 688 & 418 & 0.589 \\
\hline
\end{tabular}

HWE $P$ : $P$ value from Hardy-Weinberg equilibrium test for each study's control group.

pool the data: allelic model ( $\mathrm{G}$ allele vs. C allele), dominant model ( $\mathrm{G} / \mathrm{C}$ $+\mathrm{G} / \mathrm{G}$ vs. $\mathrm{C} / \mathrm{C})$, recessive model $(\mathrm{G} / \mathrm{G}$ vs. $\mathrm{G} / \mathrm{C}+\mathrm{C} / \mathrm{C})$, homozygote model (G/G vs. C/C) and heterozygote model (G/C vs. C/C). Subgroup analysis was performed by ethnicity. The funnel plots, Begg's test and Egger's test were used to assess the possible publication bias [7]. All statistical tests were performed by STATA 11 (Stata, College Station, Texas, USA).The level of statistical significance was set at $P<0.05$.

\section{Results}

\section{Selection process and study characteristics}

Our literature search initially identified 38 articles, PUBMED $(n=9)$, EMBASE $(n=12)$, CNKI $(n=13)$, WANFANG $(n=2)$ and CQVIP $(n=2)$. If there were duplicate researches, only the latest and most comprehensive one was included. Manual searches of article bibliographies did not identify any additional studies. Finally, six studies [8-13] (2242 cases and 2359 controls) met the inclusion criteria and were involved in the current meta-analysis. In all the populations studied, one was Korean population, others were Chinese population. All studies had a case-control design. All controls of these studies were hospital-based population and in Hardy-Weinberg Equilibrium. The main characteristics, genotype and allele distributions of these studies were summarized in Table 1.

\section{Meta-analysis results}

The main results of the meta-analysis were showed in Table 2. Totally, no significant association between the SNP rs2910164 and ischemic stroke was found in all genetic models (Table 2 and Figure 1 ), as well as in Chinese population. Whereas, in the subgroup analysis by ethnicity, significant associations were found in Korean population under allelic, homozygote and recessive model.

\section{Publication bias}

Begg's funnel plot and Egger's linear regression test were performed to assess publication biases in the included studies. The shape of the funnel plot of the association between rs2910164 polymorphism and ischemic stroke did not reveal any evidence of obvious asymmetry (Figure 2). Both Begg's and Egger's test also did not indicate any statistical evidence of publication bias under all genetic models in overall studies (Begg's test: $P=0.26$ for allelic model, $P=0.26$ for homozygote model, $P=0.85$ for heterozygote model, $P=0.85$ for dominant model, and $P=0.10$ for recessive model, respectively; Egger's test: $P=0.37$ for allelic model, $P=0.25$ for homozygote model $P=0.83$ for heterozygote model, $P=0.49$ for dominant model, and $P=0.24$ for recessive model, respectively) as well as in subgroup analysis. Therefore, there was no evidence of publication bias in the present study.
Table 2. Results of the meta-analysis for the association of rs2910164 polymorphism with ischemic stroke.

\begin{tabular}{|c|c|c|c|c|c|}
\hline Genetic model & Ethnicity & OR $(95 \% C I)$ & $\mathbf{P}$ & $\mathbf{I}^{2}$ & P-heter \\
\hline \multicolumn{6}{|c|}{\begin{tabular}{l|l} 
Allele & \\
\end{tabular}} \\
\hline & Overall & $1.051(0.881,1.254)$ & 0.582 & $75.80 \%$ & 0.001 \\
\hline & Chinese & $1.011(0.820,1.245)$ & 0.921 & $76.20 \%$ & 0.002 \\
\hline & Korean & $1.241(1.055,1.460)$ & 0.009 & & \\
\hline \multicolumn{6}{|l|}{ Homozygote } \\
\hline & Overall & $1.080(0.726,1.608)$ & 0.703 & $78.00 \%$ & $<0.001$ \\
\hline & Chinese & $0.985(0.616,1.575)$ & 0.949 & $78.20 \%$ & 0.001 \\
\hline & Korean & $1.594(1.134,2.240)$ & 0.007 & & \\
\hline \multicolumn{6}{|l|}{ Heterozygote } \\
\hline & Overall & $1.092(0.961,1.242)$ & 0.175 & $0.00 \%$ & 0.474 \\
\hline & Chinese & $1.068(0.915,1.245)$ & 0.404 & $5.10 \%$ & 0.378 \\
\hline & Korean & $1.163(0.907,1.491)$ & 0.233 & & \\
\hline \multicolumn{6}{|l|}{ Dominant } \\
\hline & Overall & $1.092(0.909,1.311)$ & 0.348 & $53.60 \%$ & 0.056 \\
\hline & Chinese & $1.051(0.842,1.311)$ & 0.661 & $56.90 \%$ & 0.055 \\
\hline & Korean & $1.259(0.995,1.592)$ & 0.055 & & \\
\hline \multicolumn{6}{|l|}{ Recessive } \\
\hline & Overall & $1.036(0.738,1.454)$ & 0.839 & $74.70 \%$ & 0.001 \\
\hline & Chinese & $0.952(0.641,1.415)$ & 0.809 & $74.30 \%$ & 0.004 \\
\hline & Korean & $1.461(1.072,1.990)$ & 0.016 & & \\
\hline
\end{tabular}

Numbers for Chinese pupolation 1564/1806 (case/control), for Korean pupolation 678/553 P: P values for combined effect; OR: odds ratio; $\mathrm{CI}$ : confidence interval; P-heter: P-value of $\mathrm{Q}$ for heterogeneity test; Random effect model was used when P-heter $<0.05$ or $\mathrm{I}^{2}>50 \%$; otherwise, fixed effect model was used. Allelic model (G allele vs. C allele); Dominant model $(\mathrm{G} / \mathrm{C}+\mathrm{G} / \mathrm{G}$ vs. $\mathrm{C} / \mathrm{C})$; Recessive model $(\mathrm{G} / \mathrm{G}$ vs. G/C + C/C); Homozygote model (G/G vs. C/C); Heterozygote model (G/C vs. C/C).

\section{Sensitivity analyses}

Sensitivity analysis was conducted to determine whether modification of the inclusion criteria affects the analysis outcome. A single study involved in the meta-analysis was removed each time to determine the influence of individual dataset to the pooled ORs for each of the studied miR-146a polymorphisms. Our results indicated that no individual study significantly affected the overall OR (Figure 3).

\section{Discussion}

Variation of the miRNA polymorphisms could affect the processing of the pre-miRNA into its mature, regulatory form, and therefore may contribute to the susceptibility to common human diseases [14]. Alteration in the expression of miRNA genes are known to contribute to the pathogenesis of stroke, including atherosclerosis, hypertension, diabetes mellitus, neuronal cell death, oxidative damage, inflammation, and edema formation [15]. However, miRNA genes 


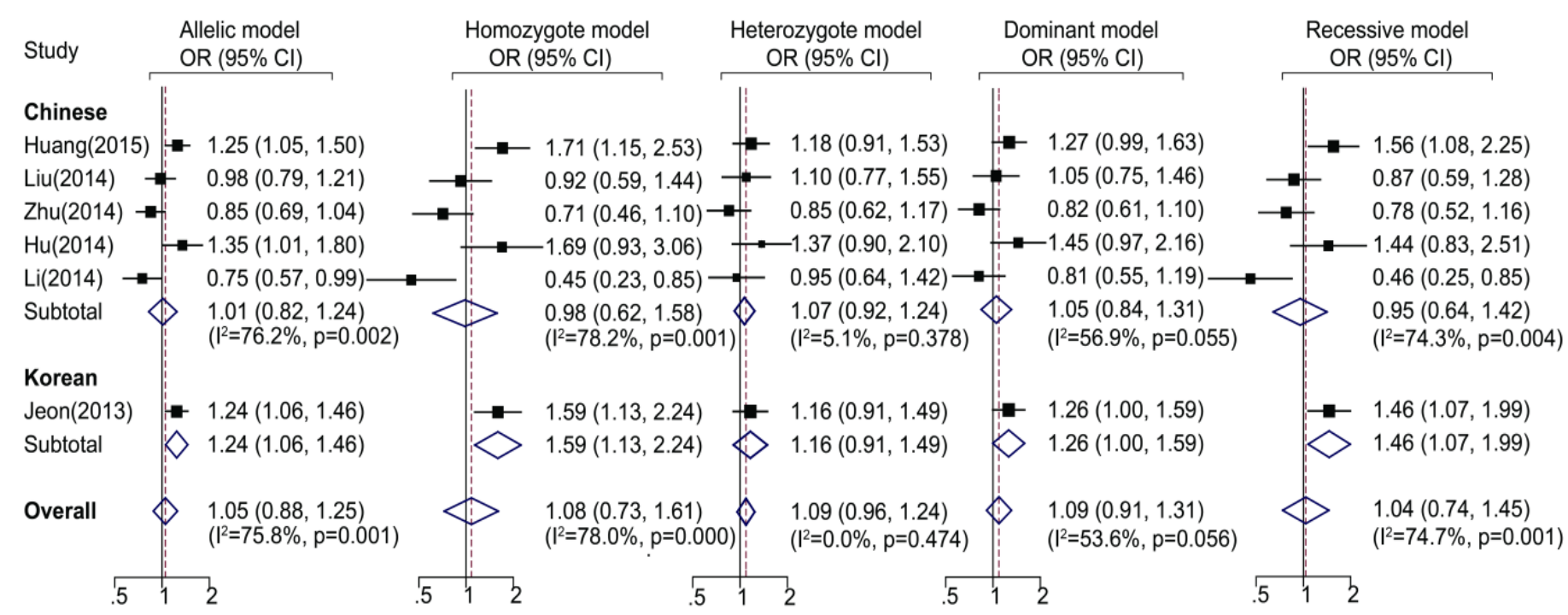

Figure 1. Meta-analysis for rs2910164 polymorphism and ischemic stroke with subgroup analyses by ethnicity. Forest plots of ORs by random effects model. Allelic model: G allele vs. C allele; Homozygote model: G/G vs. C/C; Heterozygote model: G/C vs. C/C; Dominant model: G/G +C/G vs. C/C; Recessive model: G/G vs. C/G + C/C. OR: odds ratio; CI: confidence interval.
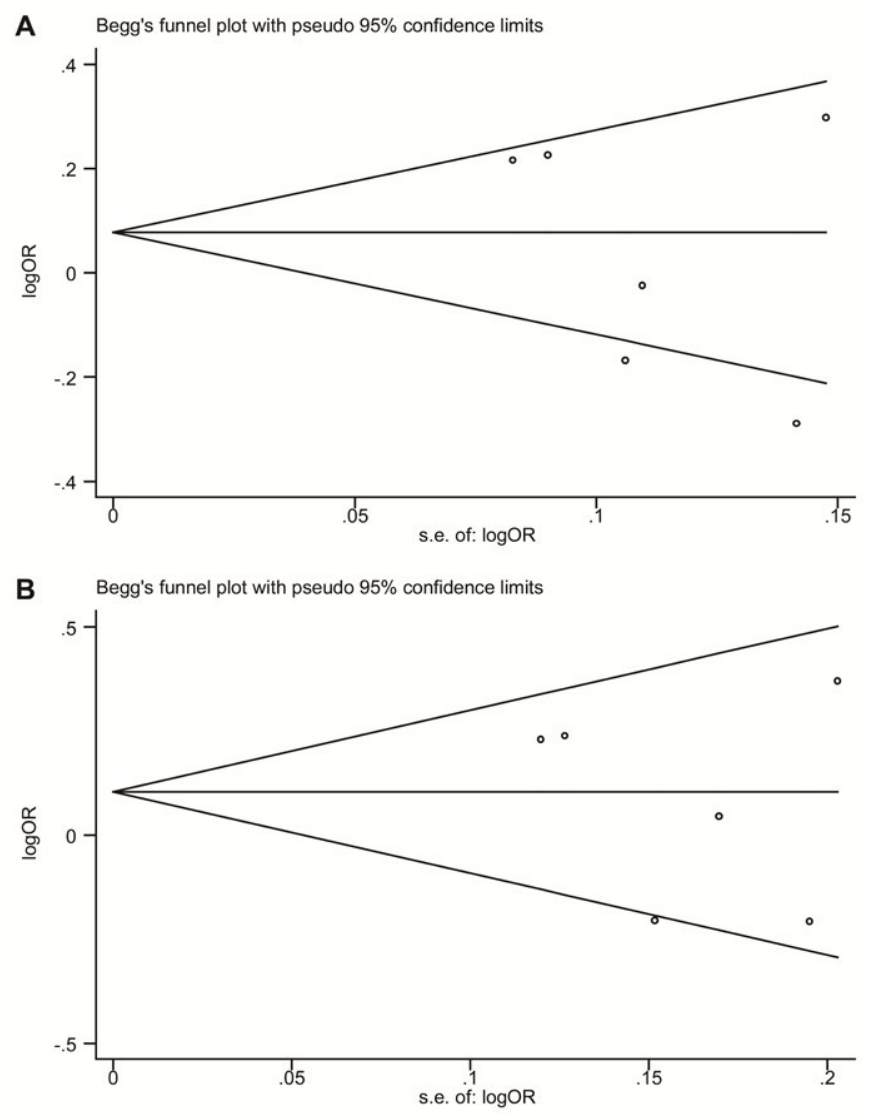

Figure 2. Funnel plots for rs2910164 on the risk of ischemic stroke in overall meta-analysis. (A) Allelic model (G vs. C); (B) Dominant model (G/G+C/G vs. C/C). s.e.: standard error; log: logarithm; OR: odds ratio. were highly conserved, the occurrence of SNPs in miRNA sequences is relatively rare [16]. There is increasing evidence that single nucleotide polymorphisms could make a significant contribution to disease susceptibility.

Several studies reported the associations between miR-146a polymorphisms and ischemic stroke susceptibility. Huang reported that subjects carrying G allele or GG genotype of rs2910164 might have increased risk of ischemic stroke [13]. Jeon found that the G allele of rs2910164 was associated with ischemic stroke in a South Korean population [12], consistent with the study of $\mathrm{Hu}$ et al. [9] in Chinese population. On the other hand, Liu et al. [11] and Zhu et al. [10] failed to find any association between the allele/genotype of rs2910164 and ischemic stroke, while Li reported a protective role of G allele[8].

The present meta-analysis showed no evidence of association of rs2910164 polymorphism with the risk for ischemic stroke. The stratified analysis based on the ethnicity also showed no associations of the rs2910164 in all genetic models in Chinese population, although it has an increased risk with ischemic stroke in Korean populations. Even though, Begg's or Egger's test indicated no publication bias and sensitivity analysis indicated that no individual study significantly altered the pooled results, this meta-analysis still has some limitations. For example, diversity in ethnicity, geographic location and genetic backgrounds of the cohort in each study could influence the results. Studies with smaller sample size may influence the statistical power, as only Chinese and Korean research data were included in our analysis and further study needs more research data from other Asian countries.

In conclusion, our meta-analysis shows that the miR-146a SNP rs2910164 is not relative to ischemic stroke in Asian population. Considering the limitations mentioned above, more studies are needed to confirm this relationship in the future. 

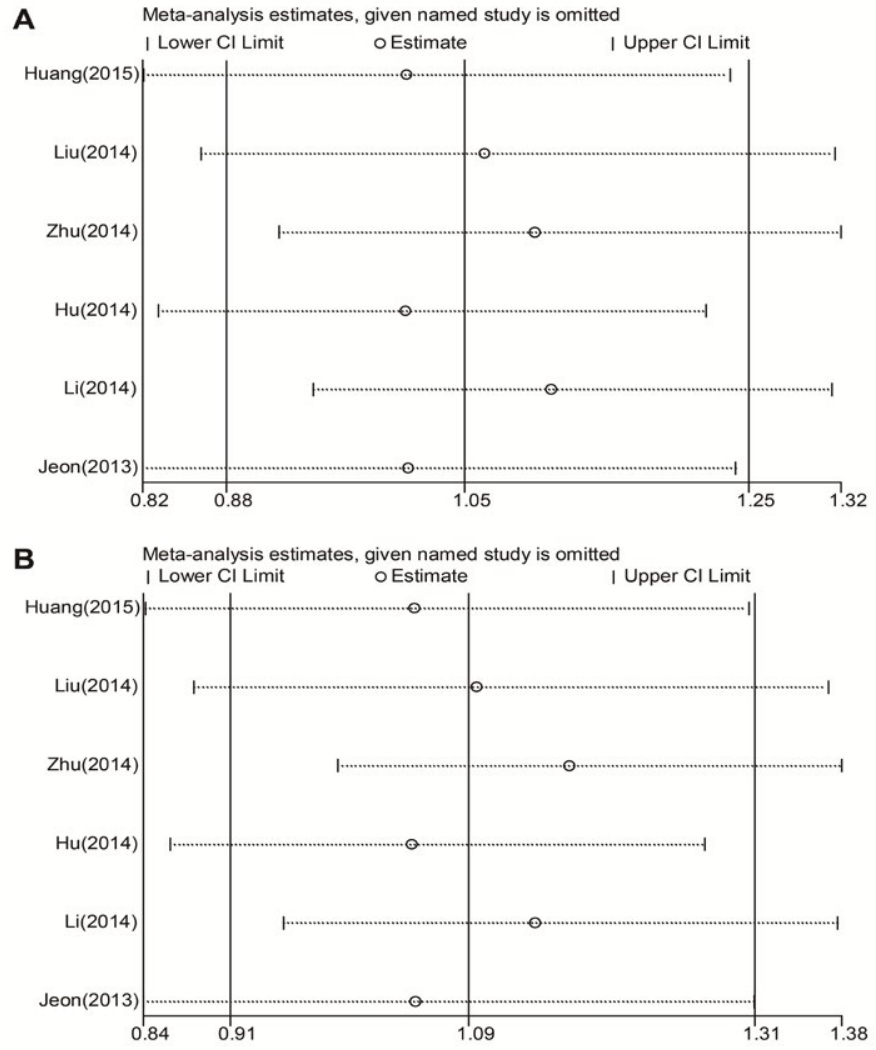

Figure 3. Sensitivity analysis for the influence of each study on pooled OR for rs 2910164 on the risk of ischemic stroke. (A) Allelic model (G vs. C); (B) Dominant model (G/G+C/G vs. C/C). CI: confidence interval.

\section{Conflict of interest statement}

All authors declare no conflict of interest.

\section{Acknowledgements}

The authors would like to thank all participants of this study.

\section{References}

1. Sturmer T, Schlindwein G, Kleiser B, Roempp A, Brenner H (2002) Clinical diagnosis of ischemic versus hemorrhagic stroke: Applicability of existing scores in the emergency situation and proposal of a new score. Neuroepidemiology 21:8-17. [Crossref]

2. Feigin VL, Lawes CM, Bennett DA, Anderson CS (2003) Stroke epidemiology: a review of population-based studies of incidence, prevalence, and case-fatality in the late 20th century. Lancet Neurol 2: 43-53. [Crossref]

3. Bevan S, Traylor M, Adib-Samii P, Malik R, Paul NL, et al. (2012) Genetic heritability of ischemic stroke and the contribution of previously reported candidate gene and genomewide associations. Stroke 43: 3161-3167. [Crossref]

4. Ebert MS, Sharp PA (2012) Roles for microRNAs in conferring robustness to biological processes. Cell 149: 515-524. [Crossref]

5. Hogg DR, Harries LW (2014) Human genetic variation and its effect on miRNA biogenesis, activity and function. Biochem Soc Trans 42: 1184-1189. [Crossref]

6. Higgins JP, Thompson SG, Deeks JJ, Altman DG (2003) Measuring inconsistency in meta-analyses. $B M J$ 327: 557-560. [Crossref]

7. Zhang Y, Zhang J, Huang J, Li X, He C, et al. (2010) Polymorphisms in the transforming growth factor-betal gene and the risk of asthma: A meta-analysis. Respirology 15: 643-650. [Crossref]

8. Li You, Zhu Jing, Ma Guo-da, et al. (2014) Association between mir-146 a gene polymorphism and lacunar infarction. Shandong Medical J 54: 1-3.

9. Hu Ya-mei, Li Shu-jian, Jiang Xiao-feng, et al. (2014) Study on the association of mir$146 \mathrm{a} c>\mathrm{g}$, mir-149 $\mathrm{t}>\mathrm{c}$ polymorphism with susceptibility to ischemic stroke. Progress in Modern Biomed 14: 5648-5650,5643

10. Zhu R, Liu X, He Z, Li Q (2014) miR-146a and miR-196a2 polymorphisms in patients with ischemic stroke in the northern Chinese Han population. Neurochem Res 39: 1709-1716. [Crossref]

11. Liu Y, Ma Y, Zhang B, Wang SX, Wang XM, et al. (2014) Genetic polymorphisms in pre-microRNAs and risk of ischemic stroke in a Chinese population. J Mol Neurosci 52: 473-480. [Crossref]

12. Jeon YJ, Kim OJ, Kim SY, Oh SH, Oh D, et al. (2013) Association of the miR-146a, miR-149, miR-196a2, and miR-499 polymorphisms with ischemic stroke and silent brain infarction risk. Arterioscler Thromb Vasc Biol 33: 420-430. [Crossref]

13. Huang S, Zhou S, Zhang Y, Lv Z, Li S, et al. (2015) Association of the genetic polymorphisms in pre-microRNAs with risk of ischemic stroke in a Chinese population. PLoS One 10: e0117007. [Crossref]

14. Ryan BM, Robles AI, Harris CC (2010) Genetic variation in microRNA networks: the implications for cancer research. Nat Rev Cancer 10: 389-402. [Crossref]

15. Tan JR, Koo YX, Kaur P, Liu F, Armugam A, et al. (2011) microRNAs in stroke pathogenesis. Curr Mol Med 11: 76-92. [Crossref]

16. Saunders MA, Liang H, Li WH (2007) Human polymorphism at microRNAs and microRNA target sites. Proc Natl Acad Sci U S A 104: 3300-3305. [Crossref]

Copyright: (C2015 Yang X. This is an open-access article distributed under the terms of the Creative Commons Attribution License, which permits unrestricted use, distribution, and reproduction in any medium, provided the original author and source are credited. 